\title{
Percepción social y Patrimonio Cultural Inmaterial (PCI): tendencias recientes y perspectivas de futuro
}

\author{
Social perception and Intangible Cultural Heritage (ICH): \\ recent trends and prospects
}

Tatiana Ramón Ramón (Associació Cultural Surc, España)

Cita bibliográfica: Ramón, T. (2022). Percepción social y Patrimonio Cultural Inmaterial (PCI): tendencias recientes y perspectivas de futuro. Disjuntiva, 3(1), 47-53. https://doi.org/10.14198/DISJUNTIVA2022.3.1.4

\begin{abstract}
Resumen
En el ámbito de la Comunitat Valenciana y del Estado Español, el reconocimientodelasfigurasdeprotección debienesculturales de carácter inmaterial ha experimentado una constante evolución a lolargo de las sucesivas reformas legislativas vinculadas, en cierto modo, a las recomendaciones internacionales de instituciones como la UNESCO, que han ayudado a conceptualizar este tipo de manifestaciones. En el ámbito de la Comunitat Valenciana, la naturaleza del Patrimonio Cultural Inmaterial (PCI), protegido desde que existen este tipo de figuras, se centra principalmente en bienes de carácter litúrgico o festivo representando una gran desigualdad frente a bienes inmateriales de otra naturaleza como saberes o técnicas. Al mismo tiempo, se han desarrollado un gran número de reconocimientos turísticos a ceremonias y actos festivos con un alto grado de reconocimiento social que desdibujan la concepción social del PCI. A través de una propuesta metodológica se pretende analizar la relación que existe entre la percepción social y el grado de protección, así como los factores y procesos que influyen en la percepción social del PCI.
\end{abstract}

\section{Palabras claves}

Bien de interés cultural; Bienes inmateriales de relevancia local; UNESCO; Patrimonio Cultural Inmaterial (PCI); turismo.

\begin{abstract}
In Spain and the Valencian Autonomous Community in particular, the recognition of the protection figures related to the intangible cultural property have undergone a constant evolution throughout the several legislative reforms linked, in a certain way, to the international recommendations of institutions. such as UNESCO, which have helped to conceptualize this type of cultural manifestations. In the area of the Valencian Community, the nature of the Intangible Cultural Heritage $(\mathrm{ICH})$, protected since these types of figures were created, have focused mainly on goods of a liturgical or festive nature, representing a great inequality compared to intangible goods of another nature such as knowledge or techniques. At the same time, many tourist awards have been developed for ceremonies and festive events with a high degree of social recognition that blur the social conception of the ICH. Through a methodological proposal it is intended to analyze the relationship that exists between social perception and the degree of protection, as well as to study the factors and processes that influence the social perception of $\mathrm{ICH}$.
\end{abstract}

\section{Key words}

BIC; BIRL; UNESCO; Intangible Cultural Heritage (ICH); tourism.

Correo electrónico de correspondencia: asoc.surc@gmail.com. 


\section{Introducción}

En los estudios sobre el Patrimonio Cultural Inmaterial (PCI, en adelante) sigue siendo una tarea pendiente el análisis de los procesos y el alcance que cobra el reconocimiento social de los bienes patrimoniales de carácter inmaterial, su conceptualización, las pautas para la adquisición de significado y los factores que influyen en su solicitud de protección, así como el análisis de los procesos de atribución de valores que se desarrollan en diferentes niveles institucionales como proponen Aldeguer y Cañizares (Aldeguer y Cañizares , 2021).

El objetivo de esta nota de investigación consiste en presentar la aproximación teórica y el diseño de la metodología que se pretende implementar para avanzar en el estudio de las consideraciones sociales sobre el patrimonio y su impacto en las figuras de protección.

Esta nota de investigación no sólo centra su atención en la evolución del marco legislativo vigente en materia de PCI como ejercicio introductorio, sino que se aproxima a la eventual reestructuración del marco legislativo en el ámbito nacional y las recomendaciones a nivel internacional. Paralelamente, la nota de investigación presenta el objetivo de un futuro proyecto más ambicioso que permita comparar la evolución de la legislación y las figuras de protección, con la concepción social que adquieren las manifestaciones del PCI en la Comunitat Valenciana.

Para tratar de favorecer una comprensión de los significados atribuidos al PCI por parte de la sociedad y estudiar, de este modo, la percepción social de los bienes inmateriales se ha optado por diseñar y aplicar, de forma exploratoria, un sondeo que permita contrastar y complementar el análisis del marco legislativo a partir de la bibliografía especializada sobre la materia.

Esta nota de investigación, por tanto, pretende indicar, de forma experimental e inicial, algunas hipótesis de trabajo que permitan avanzar en la elaboración futura de análisis más exhaustivos, no sólo en el ámbito de la Comunitat Valenciana, sino también con vocación de ser exportados a otros ámbitos geográficos y culturales.

\section{Representación social del PCI y principales figuras de protección}

Un análisis hermenéutico de la legislación en materia de protección del PCI permite constatar una evolución favorable desde la perspectiva de su consolidación normativa y la profesionalización de la misma, con un incremento de los instrumentos y figuras de protección. Sin embargo, de acuerdo con autores como Hernández-Ramírez (HernándezRamírez 2015) o Moncusí y Santamarina (Moncusí y Santamarina 2015), desde las últimas décadas del siglo XX se asiste a un proceso de inflación patrimonial producido, en gran medida, por la demanda vinculada a la terciarización de la economía y la expansión del turismo.

Esta premisa inspira, en gran medida, el proyecto de investigación que aquí se presenta, formulado con el objetivo de analizar el eventual impacto del sector turístico y la aproximación economicista que el PCI pueda tener respecto de su percepción social.

Parece evidente afirmar que el PCI ha experimentado un significativo auge cuyo inicio podemos fijar a raíz de la Convención para la Salvaguardia del PCI (UNESCO, 2003). En el caso de la Comunidad Valenciana la figura de mayor reconocimiento es la de Bien de Interés Cultural Inmaterial (BICI), y que también es la máxima figura de protección patrimonial del Estado Español, seguida del Bien de Relevancia Local Inmaterial (BRLI), que es una figura de protección autonómica para aquellos bienes con importancia local o comarcal, o a nivel del País Valenciano, pero sin la singularidad de los bienes reconocidos como BIC. Si se consulta el Inventario General de Patrimonio Valenciano se observa que de los cuarenta y tres elementos declarados BICI, solo salen de la categoría de "creencias, rituales festivos y otras prácticas ceremoniales" si tomamos la referencia que emplea la Generalitat para clasificar los Bienes Inmateriales de Relevancia Local.

Esta tendencia, en términos similares, se da precisamente con el listado de los BRLI. De los veintitrés elementos reconocidos tan sólo uno corresponde a la categoría de "Conocimientos tradicionales sobre actividades productivas, procesos y técnicas". La cifra es significativa, pues se trata de un único elemento que es compartido, además, con otras zonas del territorio nacional, así como con otros países como Francia. Grecia o Eslovenia, entre otros; a saber: la técnica constructiva de piedra en seco. En los años 2020 y 2021, empiezan a reconocerse nuevas manifestaciones que pueden 
suponer un cambio de tendencia al estar encajadas en dos categorías nuevas, siendo estas: l'Albà de l'Alcora como "Manifestaciones musicales y sonoras" en 2020, Representación de la obra El Señor Don Juan Tenorio, en Elda en 2020, o El Tribunal del Rollet del Comuner de Gràcia en Aldaia, en el año 2021.

La investigación acota el estudio al ámbito de la Comunitat Valenciana, dado que se trata del ámbito territorial cuyo rasgo diferenciador viene dado por ser un espacio que atesora un alto número de elementos reconocidos como PCI por la UNESCO en el ámbito del Estado español y donde el turismo también ha tenido un largo desarrollo y un peso importante en la economía. La Comunitat Valenciana cuenta, además, con un amplio listado de reconocimientos como BICI y BRLI, figuras de máximo reconocimiento que otorgan tanto la Comunitat Valenciana como el Estado español.

Asimismo, se ha considerado oportuno centrar la metodología en el estudio del marco legal en materia de PCI, evaluando su trayectoria histórica y la evolución más recientemente experimentada. Además, este análisis requiere una aproximación multinivel debido a la exigencia de coordinación e integración intergubernamental a la que conlleva la pluralidad de figuras de protección existentes y las Administraciones Públicas involucradas en diferentes fases de reconocimiento y gestión administrativa.

Los tres niveles de gobierno y administración que deben ser considerados llevan a analizar la Ley 4/1998, de 11 de junio, del Patrimonio Cultural Valenciano y sus posteriores modificaciones (Ley 9/2017, de 7 de abril, de la Generalitat, de modificación de la Ley 4/1998, del patrimonio cultural valenciano), los dictámenes de la UNESCO (2009, 2017) y las indicaciones, en último lugar, del Consell Valencià de Cultura (CVC), órgano consultivo del gobierno valenciano (Consell de la Generalitat) en materia de patrimonio cultural (CVC, 1997, 2004a, 2004b, 2005, 2017).

Para orientar y enmarcar la investigación se ha escogido el marco legislativo derivado de la Ley 4/1998, de 11 de junio, del Patrimonio Cultural Valenciano, como indicador del cambio social operado, a partir del análisis de sus posteriores modificaciones.

En el conjunto del Estado español, la legislación en materia patrimonial constituye una competencia que recae sobre las diferentes Comunidades Autónomas a partir de la recepción consolidada y plasmada en los diversos Estatutos de Autonomía aprobados paulatinamente tras la entrada en vigor de la Constitución Española de 1978.

La anteriormente citada ley valenciana del año 1998 describe el PCI del siguiente modo: "forman parte del patrimonio cultural valenciano, en calidad de bienes inmateriales del patrimonio etnológico, las creaciones, conocimientos y prácticas de la cultura tradicional valenciana" (Artículo 13). En relación a las clases de bienes inmateriales de interés cultural aduce lo siguiente: "aquellas actividades, conocimientos, usos y técnicas que constituyen las manifestaciones más representativas y valiosas de la cultura y los modos de vida tradicionales de los valencianos serán declarados Bienes de Interés Cultural" (artículo 26).

Como se observa, dicha descripción resulta excesivamente amplia y general, no estableciendo categorías específicas y diferenciadas para el PCI. Esta era una ley que, conforma esta orientación y alcance, estaba en consonancia con otras normas autonómicas del Estado español.

Si bien, la norma valenciana fue modificada en el año 2004 y los cambios siguen la estela marcada por las alteraciones que se estaban produciendo a nivel internacional. Autoras como Beatriz Santamarina indican como acontecimiento manifiesto la "convención para la salvaguardia del PCI" de la UNESCO, a partir de la cual el PCI quedó equiparado, en calidad y carácter, al patrimonio material y natural, al otorgarle una igualdad conceptual y, sobre todo, un cuerpo jurídico (Santamarina, 2013)

En esta ley derivada de la reforma del año 2004 se pueden apreciar varios aspectos relevantes tales como la inclusión de bienes de naturaleza tecnológica o la alusión a las "tradiciones del pueblo valenciano", en sustitución a la expresión contenida en el texto del año 1998 que conceptualizaba "la cultura y modos de vida tradicionales de los valencianos".

Esta investigación parte de la importancia de los procesos de atribución de significado que se producen a través del lenguaje. Mientras que la ley del año 1998 expresaba un cierto cariz decimonónico en una conceptualización apegada al folclore, la ley del 2004 emplea términos tales como los de "tradiciones" como lugar compartido o las de "pueblo valenciano" como un espacio vertebrador de identidad.

Esta y otras leyes autonómicas no escapan a lo que señala Martínez: "la mayoría de las leyes autonómicas que regulan el PCI están inspiradas por una noción folclórica del patrimonio etnográfico o etnológico” (Martínez, 2011). 
En el año 2007 se vuelve a efectuar una modificación de la cual cabría destacar la inclusión de la figura Espacio Etnológico como una categoría más de BIC. Esta circunstancia también alteró la clasificación de bienes inmuebles.

Si se consulta, por otra parte, lo que señala la UNESCO acerca del PCI, éste queda conceptualizado dentro de los siguientes conceptos o características:

- Tradicional, contemporáneo y viviente al mismo tiempo.

- Integrador en la medida en que nos podemos ver identificados en los valores compartidos con la nuestra y con otras costumbres de otros países y pueblos, generando un efecto cohesionador.

- Representativo, florece y pertenece a las comunidades que lo desarrollan y la transmisión intergeneracional es su garante de continuidad.

- Basado en la comunidad, solo puede ser elevado a la categoría de PCI mundial si es reconocido por la comunidad que lo crea, mantiene y transmite.

El CVC es, como se ha indicado, un órgano consultivo encargado de revisar y asesorar en materia de patrimonio cultural al gobierno valenciano y a la sociedad en general. Este organismo ha señalado recientemente varios aspectos a superar con el fin de depurar los criterios para la determinación de qué elementos cumplen con los requisitos para que un bien sea efectivamente reconocido. Así, en el año 2020 el CVC emitió un informe donde alertaba sobre los siguientes puntos:

- El criterio para evaluar si un bien es susceptible de ser protegido y reconocido debe estar avalado por especialistas que determinen cuestiones específicas como su antigüedad, permanencia e impacto, además de por su calidad, rigor histórico y “estado de conservación”, para valorar cuánto hay que permanente, cuánto de innovación y cuánto de superficial o irrelevante sin perjuicio de que debe ser valorado por la comunidad que lo desarrolla.

- Es preciso revisar, asimismo, el concepto de BIC para su mejor adecuación legislativa, con una nueva ley que se adapte a la definición, ámbitos y criterios establecidos en la Convención de la UNESCO.

- Además, se debería acompañar de un reglamento que desarrolle y facilite la misma (CVC,2020).

\section{Metodología}

Siguiendo los dictámenes de la UNESCO y las recomendaciones del CVC se podría enunciar que las principales problemáticas que presenta la conceptualización del PCI en el ámbito valenciano vendrían dadas por el exceso de elementos con un recorrido histórico de apenas medio siglo. Dentro de la Comunitat Valenciana el CVC propone, sin embargo, acotar el término de PCI para evitar una percepción errónea y desvirtuada de las manifestaciones culturales de carácter inmaterial.

En este sentido, por ejemplo, en los últimos años se han propuesto atribuir las figuras máximas de reconocimiento a posibles ceremonias festivas en las que se observa, sin embargo, que el enfoque queda ligado, en realidad, al favorecimiento de la actividad turística. Según lo anterior, esta dinámica también pretende, de algún modo, elevar la consideración social con el objetivo restrictivo de destacar un bien patrimonial sobre los de otros municipios que vendrían a atesorar manifestaciones culturales similares o idénticas.

Cabría interpretar, en este proceso, el modo en que a nivel provincial se han creado figuras de reconocimiento turístico que no son, en realidad, de protección, en un sentido conceptual, legal o profesional y técnico. Esta idea de que el reconocimiento sea turístico evidencia una pugna por destacar una manifestación sobre otra sin una evidencia técnica sobre el contenido conceptual de la correspondiente expresión o bien cultural.

Lo señalado anteriormente respecto de la proyección turística del PCI tiene su plasmación incluso en tradiciones que solo se desarrollan en un municipio, como es el caso de la Tomatina de Buñol. Una celebración que tiene su inicio en los años 50 del siglo XX y consiste en una batalla campal en la que se lanzan tomates resultantes del excedente de la producción del verano. Otro caso a destacar serían las conmemoraciones de Moros y Cristianos o la Semana Santa. 
Por otro lado, Elx y Alcoi son los municipios pioneros en el reconocimiento de manifestaciones tales como en Elx, El Misteri (UNESCO, 2001) o en Alcoi El Betlem de Tirisiti como BICI. Todo ello en un periodo de dos décadas. Esta evidencia entronca con la idea de que el reconocimiento de estas figuras de protección contribuiría a la puesta en valor de otras manifestaciones.

Para la realización de la investigación se ha optado por el diseño y la aplicación exploratoria de un sondeo que permita avanzar en la enumeración inicial de las consideraciones previamente señaladas. El sondeo se entiende como un instrumento útil aplicado con carácter previo a su aplicación en una muestra más extensa y representativa del universo de estudio considerado (población valenciana). Al mismo tiempo se ha tenido como referencia a las recomendaciones de autores y autoras Cepeda Ortega, Fontanal Merillas, Martínez Rodríguez y Cepeda Ortega (Fontanal Merillas, Martínez Rodríguez, Cepeda Ortega. 2020), quienes indican que socialmente se percibe en mayor medida el carácter material del patrimonio y que deben ser tenidas en cuenta las variables sociodemográficas y las vías de acceso al patrimonio.

Dicho sondeo ha sido difundido inicialmente siguiendo la estrategia de la bola de nieve a partir de las redes sociales de la Associació Cultural SURC, entidad promotora de la investigación. En dicho sondeo se incluyeron cuestiones tales como el grado de vinculación con los bienes patrimoniales que los encuestados consideraban que se debían proteger en su entorno cercano, ya que como la propia UNESCO indica, el PCI precisa del reconocimiento de los individuos que, a través de tales bienes patrimoniales, proyectan su vínculo entre el pasado y el futuro de la sociedad en la que viven.

El sondeo contó con veinticinco preguntas, las cuales medían en primera instancia la adscripción territorial y aspectos socioculturales. A continuación, se medía el grado de conocimiento sobre las figuras de protección del patrimonio cultural inmaterial (Patrimonio de la Humanidad, BIC, BIRL) así como el conocimiento de manifestaciones culturales de carácter inmaterial de la comunidad autónoma y del resto de comunidades, del Estado español y a nivel mundial. Paralelamente se interpelaba sobre el grado de vinculación cultural y el sentimiento de pertenencia a las diferentes manifestaciones culturales, así como por las vías de acceso o participación en su reproducción o representación. Por último, se consultaba sobre la valoración que merecía la difusión que se hace tanto desde la Conselleria como del Ministerio de Cultura.

Los resultados del sondeo indican, inicialmente, los siguientes aspectos:

- Una confusión respecto de los límites entre lo material y lo inmaterial, resultado que entronca con las apreciaciones del CVC cuando expone que deberían ajustarse las categorías del PCI.

- La mayoría de los encuestados conoce los elementos que se circunscriben a zonas próximas cuando se pregunta por el PCI en general. Este resultado reafirma el principio de proximidad que hace que un individuo o un grupo otorgue valores a un bien y los perciba o conozca como patrimonio.

- Se conoce más lo cercano y cuando el patrimonio cuenta con un reconocido puesto que, por ejemplo, la población de la Vega Baja que responde al sondeo no cita el Juzgado Privativo de las Aguas de Orihuela (JPAO), que forma parte de su comarca y, en cambio, sí indican conocer el Tribunal de las Aguas de la Vega Valencia o el de Hombres Buenos de Murcia.

- Se observa que lo que más se cita son los bienes de carácter ceremonial-litúrgico y festivo. Esto llevaría a considerar la gran cantidad de elementos reconocidos de esta misma tipología como una ventana de oportunidad para el resto de los bienes que se habrían ido retroalimentando hasta la actualidad.

\section{Consideraciones finales y propuestas de futuro}

A la luz de los análisis preliminares que se han realizado, cabría indicar que, en relación al proceso de conceptualización del PCI en el ámbito autonómico valenciano, la atribución de significado se habría visto influida por las dinámicas economicistas o de turistificación de los intangibles en la línea de lo indicado previamente por autoras como Santamarina (2017).

Esta tendencia a la turistificación del PCI se inserta en el marco de la búsqueda de la diferenciación como parte de los procesos en un escenario de globalización. Por tanto, cabría potenciar el criterio de organismos como el CVC para evitar esta desvirtuación del PCI. 
En el ámbito legislativo se observa que la Ley de Patrimonio Valenciano se ajusta a las tendencias del dinamismo social. Sin embargo, es preciso un reajuste relativo a la conceptualización del PCI, pues, tal y como advierte el CVC, es preciso establecer criterios exhaustivos para determinar qué requisitos debe cumplir un bien cultural para ser reconocido como tal.

Además, se debería incidir en los elementos que corresponden a saberes y técnicas tradicionales que cuentan con un largo recorrido histórico y reducir, así, el número de expresiones culturales con una proyección meramente de espectáculo o de ocio. Esta última idea reafirma lo mencionado anteriormente sobre el cariz folclórico que poseen todavía las leyes sobre patrimonio cultural en el Estado español. Esta visión reduccionista y estática del PCI entraría en conflicto con lo enunciado por la UNESCO y se vendría a presentar poco eficaz para la pervivencia del patrimonio común.

Por último, cabría destacar la relevancia de las principales figuras de protección existentes en el ámbito autonómico valenciano y en el resto del Estado español como herramientas útiles para la protección y potenciación del PCI. Como se ha visto tras los resultados exploratorios obtenidos en el sondeo diseñado y aplicado, la población vendría a poseer un mayor conocimiento de aquellos elementos que están reconocidos o en proceso de serlo, en contraposición con un menor conocimiento de aquellos que no lo están, incluso cuando se trata de elementos similares o idénticos.

Esta nota de investigación pretende, por tanto, poner en valor la necesidad de avanzar en el diseño de técnicas de investigación que permitan continuar ahondando en el conocimiento exhaustivo de los complejos procesos de interacción entre la atribución de significado a nivel social y la evolución legislativa de las figuras de protección.

\section{Bibliografía}

Aldeguer, B. y Cañizares, J. (2021). La atribución política de valores al patrimonio inmaterial: los tribunales consuetudinarios. Disjuntiva Crítica de les ciències socials 2 (1), 55-73.

Avrami, E., Mason, R. y De la Torre, M. (2014). Values and Heritage Conservation. Los Ángeles: The Getty Conservation Institute.

Comissió del LLegat Històric del Consell Valencià de Cultura (2020). Informe sobre el Patrimonio Cultural Inmaterial y los Bienes de Interés Cultural Inmateriales de la Comunitat Valenciana. Consell Valencià de Cultura: València.

CVC (1997). Informe sobre el anteproyecto de Ley de Patrimonio Cultural. Valencia: CVC.

(2004a). Dictamen del CVC sobre las medidas de protección del patrimonio cultural valenciano. Valencia: CVC.

(2004b). Sobre el peligro de desaparición del Tribunal de las Aguas en el marco de la amenaza de desaparición de la Huerta. Valencia: CVC.

(2005). Informe sobre la declaración de Bien de Interés Cultural Inmaterial a favor del Tribunal de las Aguas de Valencia. Valencia: CVC.

(2009). Fourth Session of the Intergovernmental Committee (4.COM). Abu Dhabi: UNESCO.

(2017). Aportes para la consolidación de la política pública sobre el patrimonio cultural inmaterial del Ecuador. Quito: UNESCO.

(2017). Informe sobre la nueva Ley de Patrimonio Cultural Valenciano. Valencia: CVC.

Fontanal Merillas, O., Martínez Rodríguez M. y Cepeda Ortega, J. (2020). La significación social del patrimonio en la Comunidad de Madrid. Aula Abierta, 49 (1), 17-24

Hernández-Ramírez, J. (2015). Turismo de base local en la Globalización. Revista Andaluza de antropología (8) 1-18.

Ley 4/1998, de 11 de junio, del Patrimonio Cultural Valenciano. 
Ley 9/2017, de 7 de abril, de la Generalitat, de modificación de la Ley 4/1998, del patrimonio cultural valenciano.

Martínez, L. (2011). La tutela legal del Patrimonio Cultural en España: valoración y perspectivas. Revista de Sociales y Jurídicas, 7, 123-150.

Santamarina, B. (2013). Los mapas geopolíticos de la UNESCO: entre la distinción y la diferencia están las asimetrías. El éxito (exótico) del patrimonio inmaterial. Revista de Antropología Social, (22), 263-286.

Santamarina, B. (2017). El Patrimonio Inmaterial en el País Valenciano: una explosión muy tangible. Revista andaluza de antropología, (12), 1-27.

Santamarina, B. y Moncusí, A. (2015). El mercado de la autenticidad: las nuevas ficciones patrimoniales. Revista de Occidente, 440 (441), 93-109.

UE (2017). Citizen engagement in the protection of cultural heritage A policy brief from the Policy Learning Platform on environment and resource efficiency. Bruselas: European Regional Development Found.

UNESCO (2003). Convención de 2003 para la Salvaguarda del Patrimonio Cultural Inmaterial. París: UNESCO.

Marzal, R. (2018). El patrimonio cultural inmaterial. El impacto de la ley 10/2015, de salvaguardia del patrimonio cultural inmaterial. Madrid: Aranzadi Thomson Reuters. 